\title{
Co-producción de conocimiento al caminar con niños, niñas y adolescentes. Miradas cruzadas entre dos investigaciones llevadas a cabo en Suiza y España ${ }^{1}$
}

\author{
Nadja Monnet ${ }^{2}$; Sara Rita Camponovo ${ }^{3}$; Zoé Moody ${ }^{4}$
}

Recibido: 14 de febrero de 2020 / Aceptado: 19 de junio de 2020

Resumen. Presentamos a continuación reflexiones metodológicas sobre la relevancia de trabajar caminando con niños, niñas y adolescentes. Este texto es un diálogo entre investigadoras de dos proyectos (uno en Suiza y otro en Cataluña) interesadas en sus recorridos diarios. Ambos equipos consideran importante no hablar en lugar de los y las jóvenes, sino permitirles participar en la investigación a través de su cuerpo y palabras.

Palabras clave: Caminar; entrevista móvil; etnografía en movimiento; investigación con, por y para niños, niñas y adolescentes; palabras de niños, niñas, adolescentes; ciudadanía.

\section{[pt] Caminhando com crianças e adolescentes em direção a uma coprodução de conhecimento. Olhares cruzados entre duas investigações realizadas na Suíça e na Espanha}

Resumo. Apresentamos abaixo reflexões metodológicas sobre a relevância do uso da caminhada no trabalho com crianças e adolescentes. Este texto é um diálogo entre pesquisadores de dois projetos (um na Suíça e outro na Espanha) interessados nos passeios diários de meninas, meninos e adolescentes. Ambas as equipes consideram importante não falar no lugar dos jovens, mas permitir que eles participem da pesquisa através do corpo e das palavras.

Palavras chave: Caminhada; entrevista em movimento; etnografia em movimento; pesquisa com meninos, meninas e adolescentes; palavras de meninos; meninas; adolescentes; cidadania.

\section{[en] Walking with Children and Adolescents to co-Produce Knowledge. Crossed Perspectives between two Studies carried out in Switzerland and Spain}

\begin{abstract}
This paper proposes methodological reflections on the relevance of mobilising walking when conducting research with children and adolescents. It intends to be a dialogue between researchers from two different projects (one in Switzerland, the other in Catalonia) focussing on the daily journeys of children and young people. Both teams consider it important not to speak for young people and to allow them to take part in research through their bodies and their words.

Keywords: Walking; mobile interview; ethnography in motion; research with, by and for children, adolescents; children and adolescent's words; citizenship.
\end{abstract}

\footnotetext{
"Exploring the way to and from school with children: an interdisciplinary approach of children's experiences of the third place", proyecto dirigido por Zoe Moody (HEP-VS), Philip D. Jaffé (CIDE) y Frédéric Darbellay (CIDE) para el Comité especializado en investigaciones interdisciplinares (CoSP-ID) del Fondo Nacional de investigación científica Suizo (FNS) [CR11I1_166050].

"Adoptions and Fosterages in Spain :Tracing Challenges, Opportunities and Problems in the Social and Family Lives of Children and Adolescents", investigación dirigida por Diana Marre del grupo de investigación AFIN, Universitat Autònoma de Barcelona, financiada por el Ministerio de Economía y Competitividad entre 2013 y 2015 [CSO2012-39593-C02-01]. Aprovechamos para agradecer aquí a la investigadora Beatriz San Román del grupo AFIN, por sus sugerencias y la última corrección del texto en castellano.

2 Antropóloga, docente en la Escuela Nacional Superior de Arquitectura de Marsella, investigadora en el Laboratorio Arquitectura/Antropología, Unidad mixta de investigación 7218 Laboratorio Arquitectura, Ciudad, Urbanismo y Entorno del Centro Nacional Francés de Investigación (LAAUMR 7218 L), Francia.

E-mail: nadja.monnet@marseille.archi.fr

3 Doctoranda FNS, Escuela Superior de Pedagogía de Valais (HEP-VS) y Centro Interfacultades en Derechos de los Niños y las Niñas (CIDE), Universidad de Ginebra, Suiza.

E-mail: sara.camponovo@unige.ch

4 Docente en la Escuela Superior de Pedagogía de Valais (HEP-VS) y colaboradora científica, grupo de investigación inter- y transdiciplinaridad, Centro Interfacultades en Derechos de los Niños y las Niñas (CIDE), Universidad de Ginebra, Suiza.

E-mail: Zoe.Moody@unige.ch 
Sumario. 1. Introducción. 2. Producir conocimiento caminando. 2.1. Espacios urbanos y andares. 2.2. Hacia una etnografía en movimiento. 2.3. Espacios, jóvenes y sus palabras. 3. Las herramientas de co-producción de conocimiento. 4. Recorrer los espacios con niños y adolescentes... 4.1 ... agiliza la palabra. 4.2 ... da espacio al silencio. 4.3. ... favorece la complicidad. 5. Conclusión y aperturas. 6. Referencias bibliográficas.

Cómo citar: Monnet, N.; Camponovo, S. R.; Moody, Z. (2020): Co-producción de conocimiento al caminar con niños, niñas y adolescentes. Miradas cruzadas entre dos investigaciones llevadas a cabo en Suiza y España, Sociedad e Infancias, $4,43-53$.

\section{Introducción}

Estudios recientes sobre Infancias (Childhood Studies) y sobre sus derechos (Children's Rights Studies) reflejan desarrollos sociales relacionados con el reconocimiento del derecho de niños y niñas ${ }^{5}$ a expresarse libremente sobre cualquier asunto que les interese (Convención de las Naciones Unidas sobre los Derechos de la Niñez, 1989). Asimismo, quienes trabajan en los ámbitos de la arquitectura y el urbanismo muestran una creciente preocupación por considerar las opiniones de la ciudadanía en la planificación de los espacios urbanos. Un número creciente de estudios (Chombart de Lauwe et al., 1976, McDonald, 2008, Poretti, 2016) han señalado la importancia de explorar la forma en que no sólo la población adulta sino también la infantil habitan el espacio público. Interesarse por los recorridos y las caminatas por la ciudad y los pueblos proporciona un acceso privilegiado (in situ) a las representaciones que las personas dan de sus prácticas cotidianas. De esta manera, quien investiga contacta también directamente con el terreno de las personas con las que dialoga e investiga, percibiendo los múltiples factores situacionales que pueden influir, de forma más o menos directa, en los comportamientos de las personas y en la relación "persona que investiga - persona investigada". Los datos así producidos están más cercanos a los eventos experimentados y, por lo tanto, tienen una mayor espontaneidad que cuando se trabaja con recuerdos o narraciones de los trayectos.

Analizamos en este artículo dos estudios que, aunque desarrollados en contextos disciplinares distintos, comparten la importancia de acercarse a las experiencias vividas por los actores sociales a través de la expresión directa de sus puntos de vista. La primera investigación se realizó con niños y niñas suizos (de 8 a 12 años) y la segunda con niños, niñas y adolescentes de Barcelona (de 10 a 20 años). Ambas exploraron la forma en que estos jóvenes ${ }^{6}$ se movían de forma independiente por las calles de su ciudad o de su pueblo, la manera en que lo vivían y de qué modo co-contribuían a su funcionamiento. El análisis de estos estudios se centrará, principalmente, en la herramienta metodológica utilizada para producir los datos necesarios para entender cómo la población más joven se adapta a un mundo pensado y estructurado por personas adultas, valorando sus prácticas y sus culturas etarias. Dichos estudios pretenden ser investigaciones no solo sobre niños y adolescentes sino con y para ellos (Morrow, 2008, Camponovo et. 2020). Un enfoque participativo que los convierte en sujetos - en lugar de objetos- de investigación, con la intención de producir conocimiento basado en sus propias experiencias con la menor influencia de la percepción y comprensión adulta. En ambos casos, caminar no ha sido el único método utilizado, es parte de un protocolo de investigación más amplio y se considera una herramienta entre otras para producir datos.

Para mostrar de qué maneras caminar puede ser una herramienta metodológica efectiva para producir conocimiento sobre infancias y adolescencias, se presentarán, en primer lugar, los marcos teóricos en los que se basaron dichas investigaciones, señalando las fuentes de inspiración de sus enfoques. Estas experiencias se pondrán luego en diálogo para mostrar las ventajas y -también- las dificultades que pueden aparecer al trabajar caminando con niños, niñas y adolescentes.

\section{Producir conocimiento caminando}

\subsection{Espacios urbanos y andares}

No pretendemos hacer aquí un repaso exhaustivo de la literatura cada vez más abundante sobre el andar por la ciudad como herramienta para elaborar una mirada crítica (en el sentido de capacidad de discernimiento y distanciamiento) para analizarla, sino señalar algunos autores que han marcado nuestra manera de abordar esta cuestión en nuestras investigaciones ${ }^{7}$. Empecemos con Colette Pétonnet, una de las fundadoras del primer Laboratorio de Antropología Urbana en Francia, quién insistió en la importancia de interesarse por el movimiento continuo de los cuerpos en la

Para no sobrecargar el texto y a pesar de reconocer la importancia de evitar términos sexistas en el lenguaje, el consejo editorial nos pidió utilizar el masculino cuando se habla conjuntamente de chicas y chicos, hombres y mujeres. Así conforme a las reglas gramaticales de la lengua española utilizaremos los plurales en masculino para hablar de ambos sexos de manera genérica. Se procedió de la misma manera en singular. Cuando se conserva el femenino significa que caracteriza a personas específicas.

En el texto, cuando se utilizará el término "jóvenes", se hace referencia conjuntamente a la niñez y adolescencia.

Para más detalle sobre los vínculos entre ciudad, arquitectura y maneras de andar por la ciudad, referirse, entre otros, a Berenstein (2006, 2012). 
ciudad. Lo considera constitutivo de la urbanidad moderna al igual que el anonimato (Pétonnet, 1987). Pocas veces esta autora está mencionada en las genealogías de los investigadores que utilizan el andar como herramienta metodológica, aunque fue ella, sin duda, quien inició el método de los itinerarios (Petiteau y Pasquier, 2001) y los recorridos comentados (Thibaud, 2001), ampliamente aplicados y luego teorizados en el laboratorio pluridisciplinar CRESSON de la Escuela de Arquitectura de Grenoble. En su capítulo "El Casco Antiguo" (Pétonnet, 2002 [1979]: 45-61), Pétonnet mostró la importancia de salir y pasear con las personas con las que trabajaba para contrastar los resultados de sus conversaciones con ellas. Después de una entrevista con una señora en su casa, quien le describió un vecindario transformado en el que ya no se ubicaba, donde todo se había modificado y la sociabilidad estaba devastada, salieron a caminar juntas un rato. Colette Pétonnet se sorprendió entonces al ver a su interlocutora saludar a varias personas, detenerse para enterarse de las novedades del vecindario, comprar, etc. La poca vida social descrita anteriormente estaba lejos de ser nula como la entrevista lo hubiera podido dejar intuir.

Al mismo tiempo, Michel De Certeau (1980), basándose, entre otros, en el trabajo de Jean-François Augoyard (1979), también puso énfasis en la fuerza de los pasos, destacando su capacidad de transformación y su potencial cuestionador de las configuraciones espaciales. Los artistas y urbanistas hablarán más tarde de "líneas de deseo" para describir las iniciativas peatonales y las de los conductores de vehículos ligeros (bicicletas, ciclomotores, etc.) que abandonan los caminos planificados, abriendo nuevas vías marcadas en el suelo con sus frecuentes pasajes, un sitio que puede revelarse al observar esas marcas. El primer número de Clara, una revista belga de arquitectura, fue dedicado, precisamente, al andar. En él, Judith Le Maire (2013) insistía en que caminar es una práctica recurrente entre arquitectos y urbanistas. A su juicio, esta "tradición" se habría originado a finales del siglo XIX en la práctica y los escritos de Patrick Geddes. Esta figura fundadora del urbanismo participativo (De Biase, et al., 2016) solía implicar, a través de su caminar, a las personas que habitaban el espacio sobre el que iba a intervenir, por considerarlas expertas en el mismo. Andar les permitía involucrarse en los asuntos de la ciudad, lo que no significa que caminar haga la participación ni que la participación requiera de un andar. Sin embargo, estas dos prácticas parecen a menudo encontrarse en los procesos participativos (Le Maire, 2013: 147). Geddes fue un pensador que anticipó muchas de las cuestiones actuales de los debates sobre urbanismo, al adoptar una perspectiva holística e interdisciplinar, que consistía en ver, tocar, sentir, estar en y estar con. Un enfoque y manera de proceder que insiste en el making (hacer), muy similar a las propuestas del antropólogo Tim Ingold $(2017,2018)$.

En el enfoque de Geddes, caminar se convierte en la contraparte esencial del conocimiento libresco. Actualmente, podríamos decir que estar in situ es la contraparte necesaria no solo del conocimiento comunicado por los libros, sino también de las visitas virtuales a un lugar, para así experimentar las discrepancias entre lo que uno entiende al navegar con Google Maps o Streetview y lo que siente al recorrerlo caminando o en coche. En los procesos participativos a lo largo del siglo XX, los discípulos (arquitectos y planificadores urbanos) de Geddes utilizaron -y siguen utilizando regularmente- el andar como herramienta para impregnarse y desvelar las dinámicas del sitio donde van a proyectar un nuevo edificio y/o cambiar configuraciones espaciales. Suelen también utilizar los recorridos guiados para dialogar sobre sus intenciones. El paso de las personas transeúntes se vuelve un valor para mesurar los lugares, el caminar una herramienta privilegiada que se complementa con otros métodos: el análisis de discursos, dibujos, maquetas, etc.

Utilizando el paso como medida, caminar permite analizar las distancias y los tiempos de los desplazamientos peatonales (el número de pasos, su ritmo, la frecuencia de los itinerarios), así como articular espacialidades y temporalidades. Esta producción de conocimiento se lleva a cabo conversando con los usuarios (residentes o no) de los lugares. Las personas participantes en la investigación acompañan a otras al lugar en cuestión para explicarles lo que saben del lugar, poniendo en evidencia su complejidad y explicando los futuros proyectos. "A través del recorrido guiado, [los urbanistas y arquitectos] 'muestran' el sitio de una manera particular y múltiple. Exponen el resumen de la investigación, la completan y la ordenan” (Le Maire, 2013: 134 ${ }^{8}$ ). Andar se convierte en una herramienta, no solo para comprender y analizar la dinámica urbana, sino también para organizar e intercambiar conocimientos.

\subsection{Hacia una etnografía en movimiento}

Esta manera de proceder converge con una cierta etnografía urbana promovida en Francia por Isaac Joseph (1998) y en España por Manuel Delgado (2003) seguida, entre otros, por Diana Arias (2017) o Monnet (2013). Para Manuel Delgado, los espacios públicos son los lugares predilectos de la urbanidad, a la que define "básicamente, como una posibilidad pura de unión, la distancia entre dos puntos, una práctica de lugares que crea sociedades entre ellos, pero que no da como resultado un lugar sino una forma de pasar. [En ellos, se producen reuniones de desconocidos] unidos por la indiferencia, el anonimato y otras películas protectoras, permanentemente expuestos a los imprevistos, al mismo tiempo que cubiertos, camuflados" (Delgado, 2004: 59).

Para captar el movimiento de esa sociedad en construcción y en constante negociación consigo misma, Manuel Delgado propuso que quien etnografíe se asemeje a las personas transeúntes, a los flâneurs. Se trata de investigar en movimiento. La etnografía en contexto urbano se convierte en un encuentro activo con la urbanidad. Para revelar lo urbano, esa realidad social reconstruida por el pensamiento, la convivencia de una época determinada, el etnógrafo se transforma en una especie de espigador de huellas que permiten entender de qué está constituida la vida urbana.

Todas las traducciones de los textos en lenguas extranjeras en la bibliografía son nuestras. 
Se diferencia de quien solo transita por la atención que presta a los detalles y a la calidad de los flujos o por la importancia que da a las agitaciones urbanas que ocurren ante sus ojos en la vía pública.

Recorrer la ciudad permite percibir límites, fronteras y volver a coser territorios que los primeros trabajos de antropología urbana tendieron a separar, creando entidades de investigación comparables a las que solían encontrar en sus trabajos de campo en tierras "exóticas". Quien investiga se adentra así en las "texturas" o las "mallas" formadas por los caminos de quienes tejen vínculos entre sí y con su entorno a través de sus tránsitos cotidianos (Ingold, 2013). Andando por las calles de una ciudad, las personas se cruzan, se codean, ignorándose cortésmente o reuniéndose explícitamente para encontrarse o, a veces, para explorar lugares juntas. En la ciudad, caminar es una forma de contactar con personas conocidas y desconocidas, establecer relaciones más o menos profundas con otros usuarios de la calle y otros espacios atravesados. Sandrine Depeau (2001) explicaba que estas ocasiones son esenciales para el buen desarrollo de los niños. David Le Breton (2015) et al. (2012), por su parte, ha enfatizado ampliamente los beneficios psicológicos, cognitivos y sociales de caminar sobre quienes lo practican.

\subsection{Espacios, jóvenes y sus palabras}

Distintas disciplinas (sociología, antropología, geografía, psicología) se han interesado en las experiencias y rutinas de los niños y adolescentes en los espacios urbanos. Estos estudios, centrados principalmente en las dimensiones geográficas y espaciales, han señalado la naturaleza porosa del espacio entre las dos esferas de influencia en la infancia: el hogar y la escuela (Herrador-Colmenero et al., 2017), una porosidad que resalta el hecho de que el espacio no es una entidad claramente delimitada. Por el contrario, sus límites borrosos favorecen los intercambios recíprocos entre el individuo y los diferentes espacios que cruza, lo que, a su vez, contribuye a su desarrollo psicológico y social (James y Prout, 1997).

Sarah L. Holloway y Gill Valentine (2000) han destacado la existencia de un fuerte vínculo entre la niñez y su entorno. Otros equipos de investigación (Burdette et al., 2004; McDonald, 2008; Sallis et al., 2000; Veitch et al., 2008) se han centrado en la libertad de los niños en los espacios que habitan diariamente (como la escuela, el hogar y el vecindario) y la forma en que pasan su tiempo en dichos espacios. Otros estudios han focalizado su atención en el peso que las amenazas de peligro (accidente, secuestro, etc.) o las distancias a recorrer tienen en la decisión de padres y madres de permitir (o no) a sus hijos e hijas transitar por la ciudad sin control adulto (Fyhri y Hjorthol, 2009; Matthews et al., 2000; Pooley et al., 2005). Esta amplia gama de investigaciones se ha centrado en datos emergentes de observaciones participantes en lugares identificados y claramente delimitados o en las narrativas de personas adultas (padres, madres, docentes, etc.). Pocos datos son los que se han recogido directamente de los propios niños y adolescentes en tanto que usuarios de esos lugares.

Las dos investigaciones presentadas a continuación coinciden en el interés de construir dispositivos con los sujetos, asumiendo su papel crucial, incluso primordial, en la producción de datos. Ambas han dado mucha importancia a escuchar la palabra de los actores directamente implicados en la investigación, en nuestro caso, niños, niñas y adolescentes. Su participación activa en la producción de datos ha permitido un acceso directo a sus prácticas, reduciendo el impacto del filtro adulto. ${ }^{9}$ A lo largo de sus vidas, las personas adultas desarrollan su propia comprensión e interpretación del mundo que les rodea. Su lectura de la realidad de los jóvenes, basada principalmente en sus conocimientos y experiencias personales, no siempre es acorde con el contexto contemporáneo y puede diferir significativamente del de los niños y adolescentes que "observan con ojos diferentes, hacen preguntas diferentes, preguntan cosas en que las personas adultas no piensan, tienen preocupaciones distintas y tienen un acceso inmediato a la cultura etaria, la cultura de sus pares, de la que los adultos no participan" (Kellet, 2005).

Sin embargo, una simple movilización utilitaria de los jóvenes en el proceso de investigación no puede ser suficiente. Como señalan Jenna Gillett-Swan y Jonathan Sargeant (2018), el concepto de participación no posiciona a los niños como una "caja de herramientas" con la cual obtener informaciones útiles. Por el contrario, "la participación debe considerarse como un proceso en el curso del cual niños [y adolescentes también] y sus puntos de vista deben estar activamente involucrados" (Gillett-Swan y Sargeant, 2018:122). Niños y niñas son agentes expertos de su vida cotidiana, sobre la cual pueden reflexionar, desarrollar un ojo crítico, hablar y proporcionar información relevante (Willumsen et al., 2014). Potenciar su voz como actores sociales en investigaciones que les conciernen es también una dimensión de su derecho a la participación, reconocido por la Convención de las Naciones Unidas sobre los Derechos de la Niñez de 1989 (art.12).

\section{Las herramientas de co-producción de conocimiento}

Entrevistar caminando, la denominada entrevista móvil (mobile interview) de Griffin (2019) y Porter et al. (2010) en el primer proyecto, y la etnografía en movimiento o recorridos acompañados en el segundo,-también denominados go-along ${ }^{10}$ por Carpiano (2009), Kusenbach (2003) y García et al. (2012)-, es una técnica de producción de datos

\footnotetext{
Para ello se necesita superar antes otro tipo de filtro: la autorización parental que da acceso a su palabra.

A menudo, es el primer y mayor obstáculo en cualquier investigación con personas menores de edad.

10 Go-along significa literalmente "ir a lo largo de", pero que tiene la idea aquí de acompañamiento: "caminar con".
} 
utilizada por diversas disciplinas, como vimos para el urbanismo, la arquitectura y la antropología, pero también la sociología, la geografía o, incluso, el estudio de la salud. El hecho de hablar caminando da protagonismo a los lugares por los cuales se transita. Activos disparadores, se vuelven "co-ingredientes constitutivos" (Anderson, 2004) en la producción de conocimiento. Permiten una co-producción de datos situados, impulsando un conocimiento colaborativo.

La primera investigación, Explorando el camino hacia y desde la escuela con niños: un enfoque interdisciplinar sobre las experiencias de niños y niñas del tercer lugar, tenía como objetivo explorar sus experiencias y prácticas a lo largo de su trayecto, en tanto que interfaz entre los dos principales entornos de sus vidas: su casa y la escuela. Su objetivo era analizar la pluralidad de experiencias individuales y colectivas en los recorridos entre sus domicilios y la escuela, a través de las cuales tenían la oportunidad de comportarse como actores sociales y, por tanto, contribuir a la co-construcción de ese espacio intersticial.

Para reflejar la heterogeneidad de sus experiencias a lo largo de esa ruta, se escogieron nueve casos de estudio de tres regiones alpinas suizas como campo de investigación, regiones geográfica y políticamente similares, al mismo tiempo que marcadamente diversas en los ámbitos lingüístico y cultural: el cantón de Grisons (de habla alemana), el cantón de Ticino (de habla italiana) y el cantón de Valais (de habla francesa). Participaron en la investigación setenta y un estudiantes de primaria de entre 8 y 12 años, pertenecientes a tres establecimientos en cada cantón, con el objetivo de garantizar una variedad de recorridos (urbano, periurbano, rural o de montaña). Los jóvenes participantes recorrían distancias de entre 500 metros y 5 kilómetros en un mínimo de diez minutos y un máximo de media hora, solos o acompañados por amistades, caminando, en bicicleta, patinete o en transporte escolar o público.

En Suiza, en la escuela primaria, niños y niñas suelen hacer cuatro veces al día el recorrido entre casa y escuela y viceversa. El camino a la escuela se percibe como una actividad ordinaria arraigada en su vida cotidiana. Representa un momento rico en experiencias que les permiten tejer, incluso fortalecer vínculos con los demás, así como interactuar con su entorno. De manera complementaria, también constituye un espacio de liberación y divertimento que les brinda bienestar físico y psicológico.

Para producir datos sobre las experiencias y prácticas de los niños a lo largo de sus trayectos casa-escuela, escuela-casa, así como sobre sus formas de interactuar con su entorno físico y social, se empleó un enfoque metodológico llamado "mosaico". Este enfoque combina tres herramientas participativas movilizadas consecutivamente, fomentando la libertad de expresión de los participantes y la construcción de una opinión personal sobre un tema concreto abordado de diversas maneras (Clark, 2010; Trell y Van Hoven, 2010). La entrevista móvil era uno de los métodos ofrecidos a quienes participaron para expresarse sobre el camino a la escuela. El dibujo del trayecto y su narración, así como una actividad de clasificación-jerarquización de imágenes llamada Diamond Ranking (Clark et al., 2013), completaron el sistema. ${ }^{11}$

En el marco de esta investigación, cada participante recorrió el camino desde su hogar a la escuela o viceversa con un miembro del equipo de investigación. Para no afectar sus rutinas diarias, el medio de transporte era elegido por la persona que se acompañaba (a pie, en bicicleta o en patinete). Durante el trayecto se sucedieron períodos de interacción verbal -oscilando entre preguntas abiertas establecidas previamente por el equipo investigador (guía de entrevista no directiva) y preguntas espontáneas surgidas por el contexto (el aquí y ahora) - con momentos de escucha y observación de los espacios transitados. Se registró de forma rigurosa la información captada en un diario de campo para analizar, por una parte, la forma en que se construyen y evolucionan las rutinas infantiles a lo largo del camino y, por otra, para entender cómo los niños socializan con sus pares, así como con la comunidad con la que comparten ese espacio-tiempo y, finalmente, poder observar las distintas formas en que expresan su agencia.

El segundo estudio, realizado como parte del proyecto pluridisciplinar y pluri-institucional, Adopciones y acogimientos en España: desafíos, oportunidades y dificultades familiares y sociales durante la infancia y la adolescencia, tuvo como objetivo analizar lo que vinculaba - o desvinculaba- a las y los jóvenes adoptados internacionalmente a la sociedad catalana. La investigación se realizó en Barcelona con dieciocho jóvenes (de 10 a 20 años) procedentes de la adopción internacional. Una parte del proyecto se centró en aquellas trayectorias en las que se desplazan por la ciudad sin personas adultas, con el objetivo de analizar de qué manera habitaban el espacio urbano y cómo el entorno les impactaba y condicionaba sus comportamientos (Monnet, San Román y Marre, 2016a). Antes de realizar las etnografías en movimiento, trabajamos sobre el tema de la autonomía de los jóvenes en la ciudad mediante cuestionarios y entrevistas y/o grupos focales en dos escuelas públicas, una en el Eixample y otra, vecina, en Ciutat Vella, con diez estudiantes de $5^{\circ}$ y $6^{\circ}$ de primaria en la primera y veintisiete de $5^{\circ}$ en la segunda durante el curso 2013-2014. En la segunda escuela, el trabajo de campo se retomó en el curso escolar 2014-2015 con los dos grupos de $6^{\circ}$ (veintisiete estudiantes $)^{12}$ (Monnet y Arias, 2016b). Ante la dificultad de que hablaran de sus trayectos habituales y sus posibles

\footnotetext{
Al no ser el tema del artículo, no nos adentraremos aquí en detallarla. Por otra parte, señalamos que las cualidades de las entrevistas móviles para entrar concretamente en la narración y la experiencia de un itinerario cotidiano realizado por los niños, ya se señalaron en un estudio piloto realizado por Moody et al. (2005).

12 En esta segunda fase, se hizo una discusión colectiva comentando los resultados del año anterior y se volvió a pasar el cuestionario (con algunas de las preguntas modificadas en función de los primeros resultados). Se realizaron, además, cinco grupos de discusión de cuatro o cinco personas, cuatro de los cuales fueron grabados. Estos grupos focales se realizaron con una metodología participativa y de carácter lúdico con el objetivo de allanar la distancia entre el equipo de investigación y las personas participantes. Esto se hizo a través de un juego que tenía como objetivo detectar formas de percepción de la alteridad al interior del aula, relacionadas principalmente con la percepción del fenotipo o el lugar de origen en la descripción y conformación de la imagen del otro.
} 
exploraciones urbanas, inicialmente se intentó actualizar el protocolo de Jean-François Augoyard (1979), consistente en pedir a las personas dispuestas a participar en la investigación que llevaran un cuaderno para apuntar y registrar el día a día de sus movimientos, sus elementos clave, sus percepciones y sentimientos durante estos itinerarios para luego comentarlos con la investigadora durante el siguiente encuentro. Les proporcionamos libretas pero también las invitamos a usar cámaras o su teléfono móvil (para grabarse, tomar notas o fotografías), si no se sentían cómodas con la escritura. Los jóvenes con quienes trabajamos, aunque receptivos a nuestra solicitud e interesados en el proyecto, muy a menudo solo completaron parcialmente su cuaderno de campo, ya sea porque hubo malentendidos sobre los pasos a seguir ${ }^{13}$ o porque, una vez la investigadora se había ido, preferían atender otros tipos de asuntos.

Decidimos entonces acompañarlas en sus trayectos, a menudo nosotras con el cuaderno de campo en el bolsillo $\mathrm{y}$, a veces, cámara en mano. Al final de cada recorrido, se realizó un importante trabajo de escritura y organización de las imágenes producidas (fotografías y películas), lo que permitió afinar la preparación de las siguientes salidas. Las personas involucradas en el proyecto fueron seguidas al menos en dos de sus trayectorias diarias. Previamente, se establecía una cita en su casa con sus padres y/o madres, en la que se les explicaba el proyecto, se solicitaban los permisos de salida y se fijaban las citas para los recorridos. A veces se hacía ese mismo día una primera entrevista con uno o ambos padres, con el o la joven o con unos y otros conjuntamente. En todos los casos, se entrevistó, además de a los niños y niñas, a una persona adulta de la familia ${ }^{14}$. Las etnografías en movimiento, realizadas en el marco de este estudio, deben mucho al "método de los itinerarios", diseñado por Jean-Yves Petiteau (2008), aunque no lo reproduce de manera exacta, ya que la investigadora estaba sola acompañando al participante, mientras que en el protocolo de los autores mencionados, se llevó a cabo una colaboración con un fotógrafo y se grababa toda la conversación durante el recorrido. En nuestro caso, por razones técnicas, partes de los trayectos fueron filmados y otros no. En estos últimos casos, se hizo necesario registrarlos a través de fichas y de charlas entre la persona que había realizado los trayectos con los jóvenes y otro miembro del equipo que no estaba presente en el momento.

Estos itinerarios acompañados pretendían acercarse a la hodología. ${ }^{15}$ Nuestro deseo era prestar especial atención a lo que la arquitecta Paola Berenstein Jacques y la coreógrafa Fabiana DultraBritto (2008) llamaron "corpografías", es decir, atender a la relación entre los cuerpos y el entorno, ese cuerpo a cuerpo con la ciudad evocado por la urbanista y periodista Jane Jacobs (1961) cuando describe el ballet de los cuerpos que resisten a la planificación urbana funcionalista o que más recientemente destacó la fotógrafa Valérie Jouve (2015) con su exposición Cuerpos en resistencia.

\section{Recorrer los espacios con niños y adolescentes...}

\section{1. ... agiliza la palabra}

Margarethe Kusenbach señala que es difícil entender e interpretar las experiencias de las personas en un contexto dado, si se estudia solo a través de la observación: "la pura observación de un entorno no permite acceder a la percepción ambiental y la experiencia de los (demás) miembros" (2003: 461). La originalidad de entrevistar andado radica en la oportunidad de estudiar el comportamiento de las personas in situ. Al alternar preguntas previamente diseñadas con preguntas más espontáneas que surgen de las oportunidades del contexto, quien investiga tiene un acceso directo a las interpretaciones que las personas otorgan a sus acciones y las de las demás personas que encuentran en el camino. Esta combinación (caminar y entrevistar) le permite estar en contacto con la realidad de sus interlocutores: acompañarlos a lo largo de sus trayectos facilita el acceso a fragmentos de su vida diaria que se elabora en base a experiencias presentes o pasadas y emociones vividas que cambian con el tiempo y el espacio (Lee e Ingold, 2006).

Recorrer un lugar "codo con codo" desata más fácilmente la palabra. Permite alcanzar opiniones que la mera observación no cubre. Agiliza ciertos temas de conversación más difíciles de abordar en una entrevista convencional. Así, por ejemplo, un joven barcelonés de 14 años, al ver pasar un automóvil rojo rememoró su salida del orfanato. El vehículo le recordó el que lo vino a buscar para llevarlo a su familia adoptiva. La aparición del coche en el trayecto le permitió expresar sentimientos en relación a una situación que difícilmente hubiera aparecido en una entrevista convencional. Un acontecimiento en el camino convergió de pronto con su trayectoria vital. Recorrido urbano y trayectoria biográfica se entremezclan. En Suiza, Lena ${ }^{16}$ (10 años), al pasar ante un grupo de casas, recordó cuando, tiempo atrás, iba con su hermana a buscar a sus amigos con los cuales luego iban juntos a la escuela. Le gustaba com-

13 Por ejemplo, una joven nos recibió durante la segunda reunión con una tarjeta de metro de diez billetes, explicándonos que no había podido hacer mucho porque sus madres solo le compraban una tarjeta por semana. Para ella, el ejercicio no pudo llevarse a cabo porque no podía tomar el metro, el único lugar donde ella consideraba hacer "trayectos" urbanos.

14 Se entrevistaron a doce personas adultas y dieciocho jóvenes, con los cuales se realizaron entre dos y seis recorridos cotidianos. Fueron en total setenta y dos encuentros (la mayoría de ellos grabados en vídeo), cuyos resultados fueron sistematizados en fichas para poder seguir los recorridos urbanos y las trayectorias de vida de cada una de los jóvenes que participaron en la investigación. Se privilegió el punto de vista del joven, ya que es un enfoque menos documentado que la visión de madres y padres respecto a la adopción. Además del material oral grabado, se compilaron también imágenes videográficas, fotografías tomadas por los propios participantes, los SMS y Whatsapps intercambiados. La primera entrevista, previa a los recorridos, sirvió para construir conjuntamente un inventario de los recorridos y elegir aquellos que se iban a acompañar.

15 Del griego «hodo»: camino. En el mundo de la geografía anglosajona y la planificación urbana, la hodologia (ciencia de los caminos) se interesa en el estudio de los caminos y los desplazamientos en los espacios.

16 Se han cambiado los nombres de todas las personas participantes en las investigaciones para preservar su anonimato. 
partir el trayecto con ese grupo al cual los vecinos llamaban "la pequeña familia". En este caso, una parte del barrio activa el recuerdo de momentos pasados de amistad y alegría.

En el relato de las personas, la movilidad arroja una luz significativa sobre sus experiencias, al mismo tiempo que muestra cómo son algunas partes de sus vidas: "la movilidad mundana de lo cotidiano, reflejada en las narraciones individuales [...], incorpora encuentros dinámicos y encarnados esenciales con el lugar", "fomenta la conversación, la compañía y permite intercambiar sobre la comprensión de la situación" (Porter et al., 2010: 92 y 101). Cuando atravesábamos la plaza donde Joan (13 años) solía jugar sin la presencia de su madre, saludó a un hombre. Este acontecimiento permitió a la investigadora saber que Joan había sido regañado por su madre unos días antes, cuando él había hecho lo mismo en su presencia, por entablar relación con una persona desconocida. Una vez terminada la reprimenda, Joan tranquilizó a su madre al explicarle que esa persona era el abuelo del niño con el que solía jugar en la plaza. Una vez más, esta información difícilmente se hubiera podido obtener de otra manera.

Por otra parte, la entrevista móvil y los itinerarios acompañados dejan un cierto margen de libertad de respuesta a las personas participantes: incluso cuando se ha establecido previamente un guión de entrevista con los temas principales a abordar y posibles cuestiones a plantear, elementos situacionales inesperados pueden convertirse en cualquier momento en desencadenantes para nuevos temas y reorientar la conversación (Carpiano, 2009, Trell y Van Hoden, 2010). Durante la entrevista móvil con Elodie (11 años), por ejemplo, un gato negro cruzó la calle de enfrente. La niña cambió inesperadamente de tema para explicar que a menudo se encontraba con animales en el camino hacia la escuela y que le gustaba detenerse para acariciarlos: "a todos los gatos negros los llamo Sr. Midnight ... porque hay un juego con un gato que se llama Mr. Midnight y ahora amo a los gatos negros". A continuación, cambió de nuevo de tema de conversación para mostrar a la investigadora un lugar que consideraba peligroso y que debía atravesar. Como los niños están por lo general profundamente arraigados en el aquí y ahora, obtener información sobre lugares peligrosos no siempre es fácil. El contexto ayuda: mediante la asociación libre de ideas, la joven dio acceso a información que podría haber permanecido desconocida.

El contexto no solo es utilizado por el investigador como una "fuente de discusión", sino que permite también a los propios actores identificar elementos que consideran importantes. De este modo, contribuyen activamente a la co-construcción de la conversación, al llamar la atención sobre elementos que pueden escapar o resultar inicialmente de poco interés para la investigadora. El hecho de caminar transforma los espacios transitados en catalizadores de una conversación dinámica. Se vuelven fuentes de información que no se pueden explorar a través de otras herramientas de investigación (véase también Cele, 2006). En base a resultados de una investigación que explora la vida cotidiana y las relaciones culturales entre grupos de jóvenes en los espacios públicos, Nicola J. Ross et al. (2009) destacaron también que

las interacciones que tomaron lugar en el movimiento fueron dinámicas, caracterizadas por un diálogo más fluido, moviéndose de un tema a otro, volviendo a los temas anteriores, permitiendo interrupciones y pausas sin restricciones. La presión para conversar se hizo menos fuerte en estas situaciones de investigación (Ross et al., 2009: 619).

\section{2. ... da espacio al silencio}

Existen posturas discrepantes respecto al silencio que a veces se establece durante las entrevistas. Se puede percibir como una señal de dificultad, falta de interés o incluso un desacuerdo por parte de las personas entrevistadas con respecto a las preguntas formuladas o, de manera más amplia, el rechazo a dar su opinión sobre el tema planteado en la conversación. Sin embargo, cada vez más investigaciones (Losonczy, 2002, De Miguel, 2004, Mazzei, 2004, MacLure et al., 2010, Torbenfeldt Bengtsson y Fynbo, 2018) destacan el importante papel desempeñado por el silencio en las entrevistas. En estos trabajos, en lugar de percibir el silencio como un obstáculo en la realización adecuada de una investigación, lo cuestionan e incorporan al análisis. Lo consideran como una acción voluntaria del interlocutor que significa algo: "los silencios no siempre están velados, no siempre son involuntarios, pero a menudo pueden ser una elección deliberada y llena de significados que incita a no hablar" (Mazzei, 2004: 20).

Thierry (12 años) utilizó el silencio durante la entrevista móvil como una señal hacia la investigadora: no quería dar información más detallada en relación con un tema que tocaba su privacidad. Explicó que le gustaba ir a la escuela sin personas adultas porque "aquí es donde somos más íntimos. Hablo con amigos, hablamos el idioma de la escuela y no el idioma de la familia". Cuando la investigadora intentó averiguar cuáles eran los temas de las conversaciones o lo que hacían con sus amigos en ausencia de personas adultas, Thierry guardó silencio. Miró a la investigadora y soltó una carcajada: el niño usó la estrategia de la risa combinada con el silencio para mostrar a su interlocutora que había ciertas cosas que suceden en el camino pero que no quería explicarlas. La investigadora le recordó que el contenido de la conversación permanecería cubierta por el anonimato y que de él dependía la decisión de si hablar o no al respecto. Después de unos segundos de silencio, el niño precisó que, de vez en cuando, hacían tonterías y luego el silencio volvió a asentarse. La investigadora entendió que no quiere adentrarse más en el tema y cambió el curso de la charla.

Jenna Gillet-Swan y Jonathon Sargeant (2018) señalan que la contrapartida del derecho a hablar reconocido por el artículo 12 de la Convención de los Derechos de la Niñez de 1998 radica en el derecho al silencio: "la 
importancia de reconocer al niño el derecho al silencio como una opción de comunicación aceptable es esencial para apoyar los derechos del niño a expresar sus preferencias" (Gillet-Swan y Sargeant, 2018: 122). El silencio ("silencio elegido") se usa estratégicamente para indicar que no queremos dar información o ir más allá en los detalles: "Por lo tanto, ciertos silencios podrían revelar una resistencia a la revelación de pensamientos, comportamientos, conocimientos privados que deben permanecer en la esfera privada" (Masson y Haas, 2010: 9). Al igual que Thierry, Tina (10 años) utilizó su derecho al silencio para no responder a la pregunta que se le planteaba. Durante la entrevista móvil, iba acompañada de su amiga, con quien a menudo compartía sus trayectos entre la escuela y la casa. Cuando se les preguntó “¿de qué están hablando camino a la escuela?”, las dos fueron reacias a dar una respuesta. Ambas se miraron y comenzaron a reírse, y luego su amiga especificó: "Creo que no podemos decirlo" (traducción del italiano). Posteriormente, Tina explicó que, para ella, era muy importante hacer el trayecto sin la presencia de personas adultas porque así podía compartir con su amiga los secretos que no quería revelar a nadie más. En este sentido, el silencio desempeña el papel de vector de resistencia o, como lo considera Kamala Visweswaran, representa una "forma de agencia que llena el espacio entre "lo que no se dice porque es evidente" y "lo que no se puede decir" (citada por MacLure et al., 2010: 498).

Los silencios son señales de situaciones que no deseamos exponer, pero también huellas de cosas indescriptibles en un cara a cara, sin el riesgo de perder la compostura o, simplemente, situaciones olvidadas, no reconocidas, desconocidas. Es lo que destaca Jesús Miguel De Miguel (2004) investigando sobre las partes perdidas, silenciadas (missing) para completar trayectorias biográficas de personas. Su búsqueda resuena en gran medida con las situaciones de los jóvenes adoptados que no siempre saben todo de su pasado o tienen vagos recuerdos de las primeras etapas de su infancia. Anne-Marie Losonczy (2002) explica cómo el hecho de permanecer en silencio en ciertas situaciones es más efectivo que preguntar. En su trabajo con personas refugiadas que constantemente están obligados responder a preguntas hechas por funcionarios, trabajadores sociales, médicos, etc., ella ganó su confianza compartiendo su silencio durante largas horas y participando en sus actividades cotidianas antes de poder establecer un diálogo abierto. El silencio le ofreció un espacio de intercambio y de negociación para la construcción conjunta del conocimiento (o lo que la autora llama el co-savoir, el saber compartido o co-conocimiento). En ésta, los aspectos emocionales entre las personas participantes y la etnógrafa se vuelven importantes. ${ }^{17}$

El silencio permite un acercamiento en la observación mutua y ayuda a construir una relación de trabajo. Puede fomentar la complicidad entre los interlocutores. Permite explorar y ser explorada, establecer relaciones donde el cuerpo también dice y actúa. Indica también una aceptación de la lentitud que permite profundizar en las reflexiones, expresarse con gestos y otros elementos no verbales. El silencio, poblado de gestos, representa una red de significados que podemos observar y analizar.

La escucha activa, incluida la del silencio, ha sido un elemento fundamental de la metodología de los itinerarios acompañados donde la escucha y la observación son inseparables. El silencio respeta el ritmo de la interacción; señala nudos, tensiones; permite estrechar los vínculos entre los interlocutores y es un espacio de negociación sobre lo que es significativo o no para el interlocutor. Algunos momentos de silencio fueron interrumpidos por canciones o llamadas hechas a amistades u otras personas; señalando de esta manera a veces un intento de acercamiento, otras marcando más bien con esta estrategia un deseo de distancia. Caminar en silencio es más fácil que enfrentar el silencio de una conversación estática cara a cara. Estos silencios "autorizados" ayudaron a la investigadora (que deambulaba por las calles de Barcelona y sus alrededores, junto a las personas participantes o detrás de ellas para filmarlas) establecer una cierta familiaridad. A lo largo de los encuentros se instaló así una cierta complicidad, revertiendo a veces la relación asimétrica adulto / joven, como cuando uno de los niños le dijo que se estaba divirtiendo mucho con ella, que para él, ella no era realmente una adulta ya que deambulaban por la ciudad de la misma manera. Otro le pidió que no les explicara a sus padres lo que habían hecho juntos. En este caso fue un silencio pedido a la investigadora. Esta situación reveló otro tipo de silencio, el que requiere el secreto; situación que planteó cuestiones éticas a la investigadora y sobre el cual poco se habla en los debates metodológicos.

\section{3. ... favorece la complicidad}

Como acabamos de señalar, otra riqueza del andar junto con los jóvenes es el reequilibrio de las relaciones de poder entre la persona adulta que investiga y las participantes. Plenamente involucradas con sus cuerpos y sus palabras, se desarrolla una relación más simétrica, más igualitaria, entre las dos partes. Las palabras de una y otra cobran la misma importancia. Se puede discutir y posicionarse en la conversación. Personas adultas y jóvenes se convierten en socias del proyecto y lo construyen conjuntamente: "los participantes de la investigación aportan su experiencia y conocimiento en el tema y el investigador su experiencia académica y metodológica” (Heath et al., 2009: 74). Se establece de esta manera una forma de reequilibrio de posicionamiento y se promueve un compromiso más colaborativo por parte de los interlocutores: el investigador desarrolla estrategias para restaurar la simetría en las relaciones de poder con los jóvenes, sus sujetos y sus colaboradores en la investigación (Mason y Urquhart, 2001).

Caminar juntos en un contexto que es familiar para los jóvenes, reduce el desequilibrio de poder que puede surgir entre ellos y el investigador. Como indica Richard Carpiano (2009), a través de este método, el niño no es visto

\footnotetext{
Esta autora subraya también el papel importante de los malentendidos en el desarrollo de este co-conocimiento, siempre en proceso y en parte impredecible. Su aclaración permite alcanzar pistas inesperadas.
} 
simplemente como un sujeto para ser entrevistado, sino que le da un lugar más central en el proceso de investigación. Esta forma de compromiso activo muestra que ellos son verdaderos conocedores de sus experiencias y de su entorno. Sus conocimientos deben ser incorporados a la investigación para que ésta sea más coherente con su realidad y, en consecuencia, con los resultados finales de la misma.

En ambos estudios presentados aquí, los jóvenes ocuparon un lugar central y activo en el proceso de producción de datos. Desde el inicio de su participación, se les consideró sujetos competentes y esenciales para una mejor comprensión de los temas investigados. La entrevista móvil o los itinerarios acompañados nos permiten adoptar un enfoque holístico que enfatiza en las prácticas individuales y colectivas de los individuos y sus significados (Masson y Haas, 2010). Permiten alcanzar directamente el significado que los participantes dan de sus experiencias, evitando traicionarlos y acercándose al máximo a su realidad.

En el caso de los adoptados de Barcelona, la dificultad fue convencer a los jóvenes de participar al estudio y luego fidelizarlos. En su agenda, a menudo muy apretada, no fue fácil encontrar momentos que convenían a todos; luego, una vez empezado el trabajo, algunos no se sintieron cómodos con el protocolo propuesto, y lo consideraron demasiado intrusivo para ser propuesto a sus amigos. Optaron entonces por itinerarios en solitario con la investigadora cuando esto no solía ser la dinámica habitual de sus recorridos urbanos. Sin embargo, los participantes que siguieron el proceso hasta el final se sintieron cada vez más a gusto y, a veces, olvidaron que era parte de una investigación. Una madre nos preguntó qué sucedía durante estos momentos compartidos porque su hijo regresaba mucho más sereno y descansado después de estos paseos urbanos. Encontró el proceso mucho más efectivo que las sesiones con el psicólogo y le hubiera gustado que la actividad continuara.

En el proyecto "Camino hacia la escuela", las investigadoras gradualmente se fundieron en el paisaje de las entradas y salidas de las jornadas escolares. Andar con los niños les permitió formar parte gradualmente de la comunidad y participar de interacciones que no hubieran tenido lugar quedándose en una postura de mera observación no participativa. Esos intercambios de experiencias favorecieron la creación de un vínculo entre las investigadoras y los niños, que en algunos casos alcanzo confidencias que desvelaron secretos íntimos. Un participante, Lucio ( 9 años) confió a la investigadora que una vez lanzó globos llenos de agua por la ventana de una casa y luego se escapó rápidamente para que no lo pillaran. Dijo que nunca lo contó a nadie, especialmente a sus padres, temiendo un posible castigo. Tejer complicidad (un buen feeling) con la participante permite a la investigadora participar de sus experiencias y acceder a historias personales inéditas.

\section{Conclusión y aperturas}

Andar tras los pasos de niños, niñas y adolescentes produce datos socioculturales incorporados y contextualizados. Este co-conocimiento se desarrolla a través de intercambios verbales y no verbales que alteran de alguna manera las relaciones habituales entre investigadores y participantes durante el proceso de investigación: "las relaciones sociales [...] no se llevan a cabo in situ sino que se desarrollan a lo largo del camino" (Ingold y Vergunst, 2008: 1). Las sensaciones, los sentimientos, las emociones y los recuerdos se movilizan a través de los cuerpos y las palabras. Se discuten y comentan los puntos de vista. No se trata de saber quién detenta la verdad sobre uno u otro tema, se trata de entender mejor los vínculos que los jóvenes tejen con sus entornos físicos (su entorno de vida: edificios, calles, plazas y otros mobiliarios urbanos, pero también con las plantas, los animales, etc.) así como sociales (relaciones etarias, relaciones entre padres e hijos, con otros adultos, etc.).

Moverse juntos es el punto clave del proceso. Esto lleva al investigador hacia un terreno desconocido, mientras que la persona que lo acompaña se vuelve el experto. Las comodidades y las asperezas del espacio recorrido pueden convertirse en pretextos para desviar o evitar la conversación, para enriquecerla y recuperarla al revelar aspectos inesperados que son difíciles de alcanzar por otras vías. La improvisación y el bricolaje (en el sentido levi-straussiano del término) se convierten en una parte integral del protocolo de investigación, otorgando una parte activa a los jóvenes en su desarrollo y su restitución. Ante su asombro, sus preguntas y / u observaciones, son ellos quienes influyen en la selección de los temas que se abordarán, indican los centros de interés y matizan las expectativas de los investigadores.

Acompañarles en sus itinerarios diarios nos permite observar y analizar cómo negocian su lugar en la ciudad o en su pueblo y, de manera general, su lugar en la sociedad, mientras reclaman, en el sentido de Henri Lefebvre, su "derecho a la ciudad". Luego queda la pregunta abierta y no menos importante de cómo incorporar sus voces en los resultados finales de la investigación. ¿Qué co-comunicaciones de resultados deberían implementarse? ¿Podemos imaginar, a la manera de Patrick Geddes, la elaboración de recorridos comentados, elegidos y comentados por los jóvenes ciudadanos para que otros habitantes escuchen su punto de vista? Y si no, ¿cómo involucrarlos en la redacción de los informes finales y artículos que les conciernen? Este es otro tema que merecería ser debatido.

\section{Referencias bibliográficas}

Anderson, J. (2004). Talking whilst walking: A geographical archelogy of knowledge. Area, 36(3), 254-261. 
Arias, D. (2017). Etnografía en movimiento para explorar trayectorias de niños y jóvenes en Barcelona. Revista de Antropología social, 26(1), 93-112.

Augoyard, J.-F. (2010 [1979]). Pas à pas; essai sur le cheminement en milieu urbain. Tours: A la Croisée (2ª edición).

Berenstein Jacques, P. (2006). Errances urbaines: l'art de faire l'expérience de la vie; autres chemins contre la spectacularisation urbain. En P.-H. Jeudy, P. Berenstein (eds), Corps et décors urbains: les enjeux culturels des villes (pp. 103-116). Paris: L'Harmattan.

Berenstein Jacques, P. (2012). Elogios aos errantes. Salvador de Bahía: EDUFBA.

Burdette, H. L., Whitaker, R. C., Daniels, S. (2004). Parental report of outdoor playtime as a measure of physical activity in preschool-aged children. Archives of Paediatric and Adolescent Medicine, 15: 353-357.

Camponovo, S., Moody Z., Darbellay F., Berchtold-Sedooka A., Jaffé, P.-D. (2020). Une approche transdisciplinaire du chemin de l'école : les enfants comme co-chercheuses et co-chercheurs. En I. Côté, K. Lavoie, R.-P. Trottier-Cyr (eds), La méthodologie de la recherche centrée sur l'enfant: recueillir et restituer la parole des enfants et des adolescent-e-s. Canada: Université du Québec en Outaouais. (en prensa).

Carpiano, R.M. (2009). Come take walk with me: The "Go-Along" interview as a novel method for studying the implications of place for health and well-being. Health \& Place, 15, 263-272.

Cele, S. (2006). Communicating place, methods for understanding children's experiences of place. Stockholm: Almqvist \& Wiksell International.

Chombart de Lauwe, M.J., Bonnin, Ph., Mayeur, M., Perrot, M., De la Soudière, M. (1976). Enfant enjeu: Les pratiques des enfants durant leur temps libre en fonction des types d'environnement et des idéologies. Paris: Editions du Centre National de la Recherche Scientifique.

Clark, A. (2010). Young Children as Protagonists and the Role of Participatory, Visual Methods in Engaging Multiple Perspectives. Am J Community Psychol, 46, 115-123.

Clark, J., Laing, K., Tiplady, L., Woolner, P. (2013). Making connections: theory and practice of using visual methods to aid participation in research. Newcastle: Research Centre for Learning and Teaching, Newcastle University.

De Biase, A., Lévy A., Castrillo Romón, M.-A. (2016). Editorial. Patrick Geddes en héritage. Espace et sociétés, 167(4), 7-25.

De Certeau, M. (1999 [1980]). L'invention du quotidien. 1. Arts de faire. Paris: Gallimard.

Delgado, M. (2004). Ethnographie des espaces publics. En J. Muntañola Thornberg, D. Provansal (eds), Anthropologie et espace; champ, méthodes et pratiques. Arquitectonics. Mind, Land \&Society (Barcelona: Universitat Politècnica de Catalunya), 10, 57-63.

Delgado, M. (2003). Naturalismo y realismo en etnografía urbana. Cuestiones metodológicas para una antropología de las calles. Revista colombiana de antropología, vol. 39, 7-39.

De Miguel, J. M. (2004). La memoria perdida. Revista de Antropología Social, 13, 9-35.

Depeau, S. (2001). Urban Identities and Social Interaction: A cross-cultural analysis of young people's spatial mobility in Paris, France and Frankston, Australia. Local environment, 6(1), 81-86.

Dultra Britto, F., Berenstein Jacques, P. (2008). Cenografias e corpografias urbanas, um diálog sobre as relações entre corpo e cidade. Cadernos PPG-AU FAUFBA, ano VI, número especial: Paisagens do corpo, 79-86.

Fyhri, A., Hjorthol, R. (2009). Children's independent mobility to school, friends and leisure activities. Journal of Transport Geography, 17, 377-384.

Garcia, C.M., Eisenberg, M.E., Frerich, E.A, Lechner, K.E., Lust, K. (2012). Conducting Go-Along Interviews to understand context and Promote Health. Qualitative Health Research, 22(10), 1395-1403.

Gillett-Swan, J., Sargeant, J. (2018). Assuring children's human right to freedom of opinion and expression in education. International Journal of Speech-Language Pathology, 20(1), 120-127.

Griffin, K.M. (2019). Participatory Research Interviewing Practices with Children. En A. Eckhoff (ed), Participatory Research with Young Children. Educating the Young Child (Advances in Theory and Research, Implications for Practice) (pp. 55-73). Cham: Springer.

Kellet, M. (2005). Children as active researchers: a new research paradigm for the $21^{\text {st }}$ century? ESRC National Centre for Research Methods (en línea). http://oro.open.ac.uk/id/eprint/7539, acceso 21 de enero de 2020.

Kusenbach, M. (2003). Street phenomenology. The go-along as ethnographic research tool. Ethnography, 4(3), 455-485.

Heath, S., Brooks, R., Cleaver, E., Ireland, E. (2009). Researching young people’s lives. London: Sage.

Herrador-Colmenero, M., Villa-Gonzalez, E. ,Chillon, P. (2017). Children who commute to school unacompanied have greater autonomy and perceptions of safety. Acta Paediatrica, 106, 2042-2047.

Holloway, S.L., Valentine, G. (2000). Children's geographies and the new social studies of childhood. En S. L. Holloway, G. Valentine, Children's geographies: Playing, living, learning (pp. 1-26). London: Routledge.

Ingold T. (2011-2013). Une brève histoire des lignes. Paris: Zones sensibles. [VO en inglés, 2007].

Ingold T. (2017). Faire; Anthropologie, Archéologie, Art et Architecture. Bellevaux: Editions Dehors. [VO en inglés, 2013].

Ingold T. (2018). L'anthropologie comme éducation. Rennes: Presses universitaires de Rennes. [VO en inglés, 2017].

Ingold, T., Vergunst, L. (2008). Introduction. En T. Ingold, L. Vergunst (eds), Ways of walking: ethnography and practice on foot (pp. 1-19). Ashate: Aldershot.

Jacobs J. (2011). Muerte y vida de las grandes ciudades. Madrid: Capitán Swing [VO en inglés 1961].

James, A., Prout, A. (1997). Constructing and reconstructing childhood Contemporary issues in the sociological study of childhood. London: Routledge. 
Joseph, I. (1998). Retomar la ciudad. Medellín: Universidad Nacional de Colombia.

Jouve, V. (2015). Corps en résistance. Paris: Filigranes Éditions.

Lee, J., Ingold, T. (2006). Fieldwork on Foot: Perceiving, Routing, Socializing. En S. Coleman, P. Collins (eds), Location the Field: Space, Place and Context in Anthropology (pp. 67-86). London: Bloomsbury Academic Collections.

Le Breton, D. (2015). Elogio del caminar. Madrid: Siruela.

Le Breton, D., Marcelli D., Ollivier, B. (2012). Marcher pour s'en sortir. Toulouse: Éditions Érès.

Le Maire J. (2013). Arpenter, guider, jalonner, la marche, outil des processus participatifs en architecture et urbanisme du XXème siècle. Clara, 1, 133-148.

Losonczy, A.-M. (2002). De l'énigme réciproque au co-voir et au silence. En Ch. Ghasarian (ed), De l'ethnographie à l'anthropologie réflexive (pp. 91-102). Paris: Armand Colin.

MacLure, M., Holmes, R., Jones, L., MacRae, C. (2010). Silence as Resistance to Analsysis: Or, on Note Opening One's Mouth Properly. Qualitative Inquiry, 16(6), 492-500.

Mason, J., Urquhart, R. (2001). Developing a model for participation by children in research on decision making. Children Australia, 26(4), 16-21.

Masson, E., Hass, V. (2010). Dire et faire: l'intersubjectivité dans l'entretien de recherche. Bulletin de psychologie, 505, 5-13.

Matthews, H., Limb, M., Taylor, M. (2000). The « street as thirdspace». En S. L. Holloway, G. Valentine (eds), Children's Geographies: Playing, Living, Learning (pp. 63-79). London: Routlege.

Mazzei, L. A. (2004). Silent Listenings: Deconstructive Practices in Discourse-Based Research. Educational Researcher, 33(2), 26-34.

McDonald, N. C. (2008). Children's mode choice for the school trip: the role of distance and school location in walking to school. Transportation, 35, 25-35.

Monnet, N. (2013). Flanâncias femininas e etnografia. Redobra, año 4, no 11: 218-234. http://www.redobra.ufba.br/wp-content/ uploads/2013/06/redobra11_23.pdf

Monnet, N., San Román, B., Marre, D. (2016a). Étrangers dans leur ville; les jeunes issus de l'adoption internationale dans la ville de Barcelone. Ethnologie française, XLVII, nº 2: 335-346.

Monnet, N., Arias, D. (2016b). Se déplacer sans adultes en ville : récits d'autonomie de jeunes barcelonais. Jeunesse: Young People, Texts, Cultures (Special Issue of *Jeunesse* on Mobility), vol. 8, $\mathrm{n}^{\mathrm{o}}$ 1: 89-110.

Moody, Z., Hirschi C., Steiner E., Jaffé. P.-D. (2015). Le chemin de l'école: enquête dans une commune valaisanne. Résonances 6: $12-14$.

Morrow, V. (2008). Ethical Dilemmas in Research with Children and Young People about their Social Environments. Children's Geographies, 6(1), 49-61.

Petiteau, J.-Y. (2008). La méthode des itinéraires ou la mémoire involontaire. En A. Berque, A. De Biase, Ph. Bonnin (eds), L’habiter dans sa poétique première (pp. 102-115). Paris: Editions Donner Lieu.

Petiteau, J.-Y, Pasquier, E. (2001). La méthode des itinéraires: récits et parcours. En M. Grosjean, J.-P. Thibaud (eds), L'espace urbain en méthodes (pp. 63-78). Marseille: Ed. Parenthèses.

Pétonnet, C. (1987). L'anonymat ou la pellicule protectrice. Le temps de la réflexion VII (La ville inquiète), 247-261.

Pétonnet, C. (2002 [1975]). On est tous dans le brouillard. Paris: Ed. du C.T.H.S.

Pooley, C.G., Turnbull, J., Adams, M. (2005). The journey to school in Britain. Area, 37(1). 43-53.

Poretti, M. (2016). Enfances urbaines et politiques publiques. Regards croisés d'enfants de différents quartiers de la ville de Sion. Sion $(\mathrm{CH})$ : Centre interfacultaire en droits de l'enfant (CIDE) [informe de investigación sin publicar].

Porter, G., Hampshire, K., Abane, A., Munthali, A., Robson, E., Mashiri, M., Maponya, G. (2010). Where dogs, ghosts and lions roam: learning from mobile ethnographies on the journey from school. Children's Geographies, 8(2), 91-105.

Ross, N. J., Renold, E., Holland, S., Hillman, A. (2009). Moving stories: Using mobile methods to explore the everyday lives of young people in public area. Qualitative Research, 9, 605-623.

Sallis, J., Prochaska, J., Taylor, W. (2000). A review of correlates of physical activity of children and adolescents. Medicine and Science in Sports and Exercise, 32(5), 963-975.

Thibaud, J.-P. (2001). La méthode des parcours commentés. En M. Grosjean, J.-P. Thibaud (eds), L'espace urbain en méthodes (pp. 79-99). Marseille: Ed. Parenthèses.

Torbenfeldt Bengtsson, T., Fynbo, L. (2018). Analysing the significance of silence in qualitative interviewing: questioning and shifting power relations. Qualitative Research, 18(1), 19-35.

Trell, E.-M., van Hoven, B. (2010). Making sense of place: exploring creative and (inter)active research methods with young people. Fennia - International Journal of Geography, 188(1), 91-104. https://fennia.journal.fi/article/view/2522, acceso 21 de enero de 2020.

Veitch, J., Salmon, J., Ball, K. (2008). Children's active free play in local neighborhoods: a behavioral mapping study. Health educatioin research, 23(5), 870-879.

Willumsen, E., VegarHugaas, J., Studsrod, I. (2014). The Child as Co-researcher - Moral and Epistemological Issues in Childhood Research. Ethics and Social Welfare, 8(4), 332-349. 